\title{
Classification of nanoparticle diffusion processes in vital cells by a multifeature random forests approach: Application to simulated data, darkfield and confocal laser scanning microscopy
}

\author{
Thorsten Wagner ${ }^{a}$, Alexandra Kroll ${ }^{b}$, Martin Wiemann $^{c}$, Hans-Gerd Lipinski $^{a}$ \\ ${ }^{a}$ Biomedical Imaging Group, Dept. of Informatics, University of Applied Sciences and Arts Dortmund, \\ Emil-Figge-Str. 42, D-44227 Dortmund \\ ${ }^{b}$ EAWAG, Swiss Federal Institute of Aquatic Science and Technology, Überlandstrasse 133, CH-8600 Dübendorf \\ ${ }^{c}$ Institute for Lung Health (IBE RD gGmbH) Münster, Mendelstr. 11, D-48149 Münster
}

\begin{abstract}
Darkfield and confocal laser scanning microscopy both allow for a simultaneous observation of live cells and single nanoparticles. Accordingly, a characterization of nanoparticle uptake and intracellular mobility appears possible within living cells. Single particle tracking makes it possible to characterize the particle and the surrounding cell. In case of free diffusion, the mean squared displacement for each trajectory of a nanoparticle can be measured which allows computing the corresponding diffusion coefficient and, if desired, converting it into the hydrodynamic diameter using the Stokes-Einstein equation and the viscosity of the fluid. However, within the more complex system of a cell's cytoplasm unrestrained diffusion is scarce and several other types of movements may occur. Thus, confined or anomalous diffusion (e.g. diffusion in porous media), active transport, and combinations thereof were described by several authors. To distinguish between these types of particle movement we developed an appropriate classification method, and simulated three types of particle motion in a 2D plane using a Monte Carlo approach: (1) normal diffusion, using random direction and step-length, (2) subdiffusion, using confinements like a reflective boundary with defined radius or reflective objects in the closer vicinity, and (3) superdiffusion, using a directed flow added to the normal diffusion. To simulate subdiffusion we devised a new method based on tracks of different length combined with equally probable obstacle interaction. Next we estimated the fractal dimension, elongation and the ratio of long-time / short-time diffusion coefficients. These features were used to train a random forests classification algorithm. The accuracy for simulated trajectories with 180 steps was 97\% (95\%-CI: 0.9481-0.9884). The balanced accuracy was 94\%, 99\% and 98\% for normal-, sub- and superdiffusion, respectively. Nanoparticle tracking analysis was used with $100 \mathrm{~nm}$ polystyrene particles to get trajectories for normal diffusion. As a next step we identified diffusion types of nanoparticles in vital cells and incubated V79 fibroblasts with $50 \mathrm{~nm}$ gold nanoparticles, which appeared as intensely bright objects due to their surface plasmon resonance. The movement of particles in both the extracellular and intracellular space was observed by dark field and confocal laser scanning microscopy. After reducing background noise from the video it became possible to identify individual particle spots by a maximum detection algorithm and trace them using the robust single-particle tracking algorithm proposed by Jaqaman, which is able to handle motion heterogeneity and particle disappearance. The particle trajectories inside cells indicated active transport (superdiffusion) as well as subdiffusion. Eventually, the random forest classification algorithm, after being trained by the above simulations, successfully classified the trajectories observed in live cells.
\end{abstract}

\section{INTRODUCTION}

Transport processes of particulate structures inside cells form one major backbone of cellular functions. The ways small objects may take inside cells provide insights into the mechanical properties of the local surroundings, ${ }^{1}$ can unravel nanoparticle cell entry mechanisms ${ }^{2}$ or can give insights into the movements of single proteins in the cell membrane or cytoplasm. ${ }^{3}$ In all these cases, the trajectories of single objects need to be carefully analysed. Basically, these analyses allow categorizing the observed trajectories into normal diffusion, superdiffusion or 
subdiffusion. Normal or "free" diffusion takes place when the particle movements occur completely unrestricted. Superdiffusion is an active process and may become evident when small corpuscles such as vesicles are transported by molecular machines along microtubules. ${ }^{4,5}$ Subdiffusion is a confined or anomalous diffusion observable for trapped particles or particles whose diffusion is confined by cytoskeletal elements. ${ }^{6}$

Arcizet et $\mathrm{al}^{7}$ classified the trajectories in active and passive tracks based on two features only: The exponent of a fitted power distribution and the standard deviation of the angle correlation function. By applying this method to sub-trajectories using a moving window multiple passive or active parts in a single trajectory could be distinguished. Huet et $\mathrm{al}^{8}$ calculated the diffusion coefficient, the curvature of the mean squared displacement curve and the asymmetry of the trajectory. By using six different thresholds they classified the trajectories into constrained, directed and stalled motion categories. In addition to Arcizet et al. the approach of Huet could also be applied to sub-trajectories using a moving window. Only a single parameter for classification was used by Suh et al. ${ }^{9}$ The so called "Relative Change" (RC) was defined as the ratio of the probed diffusion coefficient and a reference diffusion coefficient. The RC-value was evaluated for two different time lags and classified into the categories diffusive, subdiffusive and active using confidence intervals of the $\mathrm{RC}$ value for normal diffusion. Unfortunately the confidence interval has to be estimated for each track length which complicates a general application. Monnier et al. ${ }^{6}$ used a Bayesian approach and is able to distinguish 7 different diffusion models. Contrary to the approaches of Arcizet and Huet the method is unable to distinguish multiple modes of diffusion in a single trajectory. Also its performance decreases in case that different particles display heterogeneous modes of diffusion, which alltogether hampers its general applicability.

Since the available methods share some practical limitations, here we propose a method which successfully distinguishes between normal, super- and subdiffusive trajectories without the need for a knowledge-based configuration. To this aim we estimated multiple features of the trajectories such as fractal dimension, elongation, or the ratio of long-time / short-time diffusion coefficients. These features were used to train a random forests classification algorithm. To show that these features suffice for a classification of trajectories we validated the approach by applying it to simulated data, as well as to those obtained with darkfield (DFM) and confocal laser scanning microscopy (CLSM).

\section{MATERIAL AND METHODS}

\subsection{Imaging equipment and data acquisition}

\subsubsection{Dark field microscopy}

Hamster fibroblasts (V79, cultured in DMEM supplemented with $10 \%$ fetal calf serum) were grown to ca. $50 \%$ confluence on a coverslip (20x20 $\left.\mathrm{mm}^{2}\right)$. To set up a live observation chamber, two strips of cover slip glass $\left(5 \times 20 \times 0.17 \mathrm{~mm}^{2}\right)$ glued on a glass slide served as spacers, onto which the cell laden cover slip was mounted upside down. The chamber was rinsed $(0.1 \mathrm{ml} / \mathrm{min})$ with Krebs Ringer solution containing $2 \mathrm{mM}$ glucose (KRPG). The chamber was mounted onto the stage of an Olympus BX51 microscope heated to $37^{\circ} \mathrm{C}$. The cell containing optical plane was illuminated with a CytoViva ${ }^{T M}$ darkfield oil condenser. To load cells with nanoparticles under optical control the chamber was perfused with a low concentration $(5 \mu \mathrm{g} / \mathrm{ml})$ of PVP-coated gold nanoparticles (size: $50 \mathrm{~nm}$, Nanocomposix) added to the superfusate. Image series were taken with a color CCD camera (PCO VGA) at a constant frame rate of 30 FPS.

\subsubsection{Confocal laser scanning microscopy}

Confocal laser scanning microscopy (CLSM) was used to record nanoparticle movement outside and inside V79 cells. V79 cells were seeded at $2 \times 10^{5}$ cells $/ \mathrm{mL}$ in $5 \mathrm{~mL}$ medium in glass-bottom petri dishes (CELLView No. 627860, Greiner, 35x10 mm, tissue culture treated). After $18 \mathrm{~h}$, medium was exchanged and cells were imaged using a Leica SP5 setup equipped with a $\mathrm{CO}_{2}$ gassing and temperature control unit. Cells were thus maintained at the same conditions as during culturing. Samples were scanned with the $488 \mathrm{~nm}$ laser line and reflected light was detected from 483-493 $\mathrm{nm}$ along with the bright-field image (water immersion objective $(63 \mathrm{x} / 0.9)$ ). Data was recorded continuously in a region of $41 \times 41 \mu \mathrm{m}$ at a scan rate of $1400 \mathrm{~Hz}$ resulting in a framerate of $94 \mathrm{~ms}$; the pinhole was set to $600 \mu \mathrm{m}$ (5.778 $\mathrm{mm}$ layer), image resolution was 256x256. After several minutes of recording, control medium was replaced carefully with medium containing $10 \mathrm{\mu g} / \mathrm{mL}$ citrate-coated gold nanoparticles (size:

Proc. of SPIE Vol. $9887988722-2$ 
$50 \mathrm{~nm}$, Nanocomposix). After imaging nanoparticles in the presence of V79 cells, samples were transferred back to regular culture conditions and imaged again after $24 \mathrm{~h}$ as described above. Recorded data was exported in *.avi format without overlaying channels.

\subsubsection{Nanoparticle Tracking Analysis}

Measuring the hydrodynamic diameter of nanoparticles by tracking light scattering nanoparticles in videos is an increasingly used technique. NP suspensions are illuminated by a laser and an ensemble of light scattering particles is recorded over time by a CCD camera. The Brownian motion of each single nanoparticle is tracked using image analysis techniques. For each trajectory the mean squared displacement of the NP is estimated. The corresponding diffusion coefficient is calculated and converted into the hydrodynamic diameter using the StokesEinstein equation. Finally, the calculated diameters are used to estimate the size distribution. Importantly, this method relies on low particle concentrations (ca. $5 \times 10^{8} / \mathrm{ml}$ ) at which unrestrained particle diffusion takes place. As unrestricted diffusion is expectedly rare inside cells, we classified trajectories of videos of previous experiments with 100nm polystyrene nanoparticles (Kisker-Biotech, PPs-0.1) which were collected with a NanoSight ${ }^{T M}$ LM10 system equipped with a LM14 green $(535 \mathrm{~nm})$ laser module and a cooled Andor camera (Andor-DL-658- OEM).

\subsection{Simulation}

All simulations were carried out in Java. ${ }^{10}$ Several types of normal-, sub- and superdiffusion were simulated. Therefore we choose the track lengths $\mathrm{N}$ of 60 steps (short), 180 steps (middle) and 540 steps (long) which corresponds to a trajectory duration $\mathrm{T}$ of $2 \mathrm{~s}, 6 \mathrm{~s}$ and $18 \mathrm{~s}$ given a timelag between two frames of $\Delta t=1 / 30 \mathrm{~s}$. As diffusion coefficient we choose to use $D=9.02 \times 10^{-14 \mathrm{~m} / \mathrm{s}^{2}}$, which corresponds to the diffusion coefficient of a 50 $\mathrm{nm}$ particle at $22^{\circ} \mathrm{C}$.

\subsubsection{Normal diffusion (ND)}

For the normal or "free" diffusion we randomly choose a start position, a random direction, then draw a random steplength from the steplength density ${ }^{11}$

$$
F_{d}(u)=2 u /(4 D \Delta t) \exp \left(-u^{2} /(4 D \Delta t)\right)
$$

and finally add the displacement to the last position. The procedure to generate $\mathrm{K}$ steps could be formalized as follows:

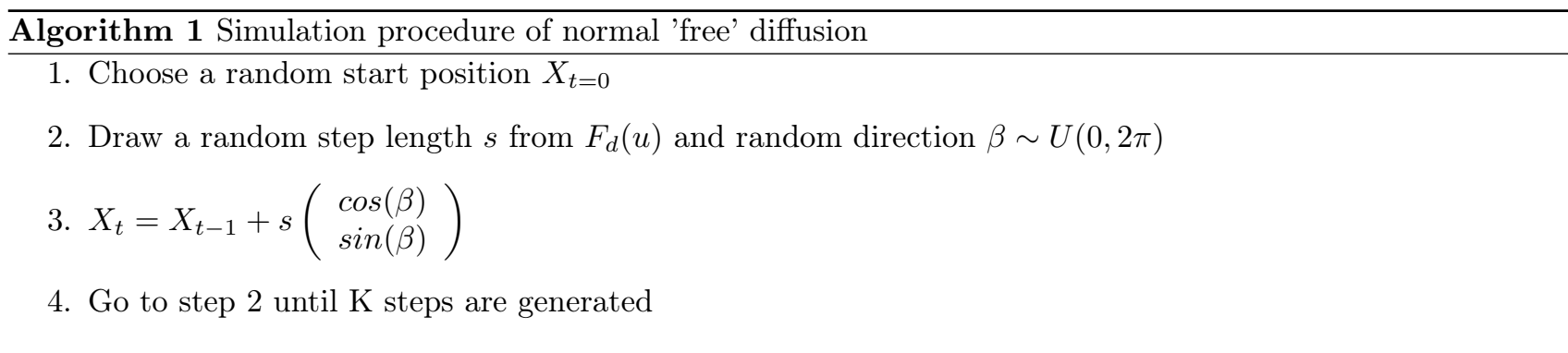

\subsubsection{Subdiffusion}

If diffusion is hindered by a reflective boundary or obstacles subdiffusion occurs. The dependence of the mean squared displacement $\zeta^{2}(\delta)$ of the timelag $\delta=n \Delta t$ (with $n \in \mathbb{Z} \geq 1$ ) is no longer linear and follows a power law $\zeta^{2} \propto D \cdot \delta^{\alpha}$ with $\alpha<1$.

\section{Confined diffusion (CD)}

We assume that a particle starts at the center of $2 \mathrm{~d}$ circular reflective boundary. To simulate a single step of a confined diffusion 100 substeps were generated by drawing random steplength from $F_{d}(u)$ with $\Delta t^{\prime}=\Delta t / 100$ and random direction and then update the latest position (of the substeps) only if the distance is smaller than 
the radius of the circular reflective boundary measured from its center. For the simulation of the confined diffusion we chose the radius in dependence of track length to ensure that the probability of interaction between the boundary and the particle is constant over all track lengths. Therefore the radius is chosen in that way that the distance traveled during the track duration $\mathrm{T}$ is larger than the radius with a probability $p_{0}=90 \%$. Reformulating the cumulative distribution function of $F_{d}(u)$ we get the following function for the determination of constant interaction radius $(\mathrm{CIR})$ :

$$
C I R_{p_{0}}=\sqrt{-\ln \left(p_{0}\right) 4 D T}
$$

This leads to a radius of $0.276 \mu \mathrm{m}, 0.477 \mu \mathrm{m}$ and $0.827 \mu \mathrm{m}$ for track durations $2 \mathrm{~s}, 6 \mathrm{~s}$ and $18 \mathrm{~s}$ respectively. Finally, the procedure to generate a confined diffusion trajectory with $\mathrm{K}$ steps is as follows:

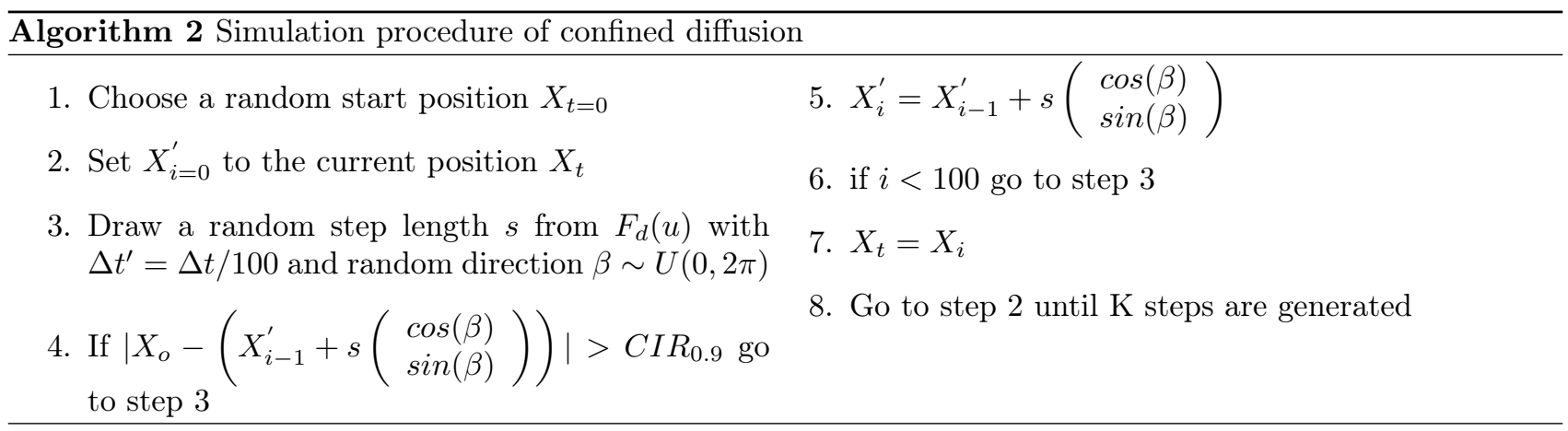

Anomalous diffusion (AD)

The simulation of anomalous diffusion is similar to the confined diffusion. However, instead of a single reflective circular boundary there are multiple randomly placed reflective obstacles in the neighborhood of the particle and a substep is rejected when the candidate position is inside an obstacle. To generate a 10x10um neighborhood we used the following procedure:

Algorithm 3 Simulation procedure to generate a neighborhood with obstacles for the simulation of anomalous diffusion.

1. Choose a random obstacle radius from $N(\mu=0.5 \mu \mathrm{m}, \sigma=1 / 12 \mu \mathrm{m})$

2. Place an obstacle at a random position in the neighborhood

3. Estimate the interaction probability for the neighborhood

4. If the interaction probability is below 0.95 , go to step 1 .

The interaction probability is estimated using the Monte Carlo method by generating $10^{5}$ circles with radius $C I R_{0.9}$ and count the ratio of circles which overlapping with obstacles. Figure 1 shows the neighborhood for the different track lengths. The fraction of volume which is excluded by obstacles is $95 \%, 93 \%$ and $65 \%$ for trajectories with 60 steps, 180 steps and 540 steps respectively.

\subsubsection{Superdiffusion}

To simulate active transport (AT) / flow processes we define a velocity $v$ and because active transports do not always follow a straight line we additionally define the angular velocity $v_{\text {rad }}$ for the change $\Delta \beta$ of direction $\beta$. The positions are updated as follows $X_{t}=X_{t-1}+v \Delta t\left(\begin{array}{c}\cos \left(\beta_{t}\right) \\ \sin \left(\beta_{t}\right)\end{array}\right)$ where $\beta_{t}=\beta_{t-1}+\Delta \beta$ with $\Delta \beta= \pm v_{\text {rad }} \Delta t$. To generate trajectories of higher complexity where normal or confined motion is combined with flow, we generate two trajectories of the same length: one active and diffusion trajectory and then add the corresponding positions. 


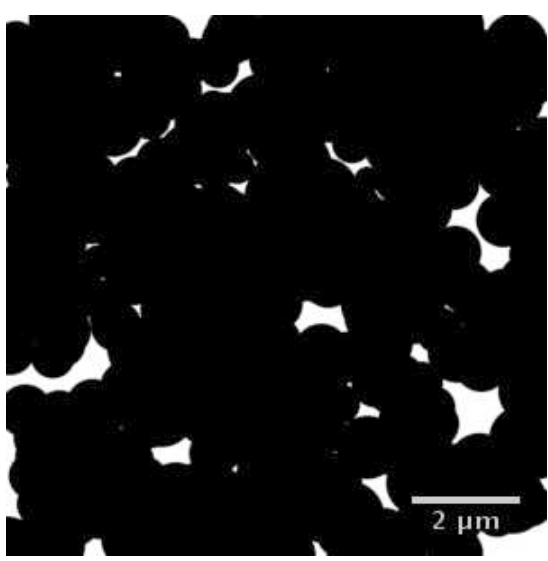

(a)

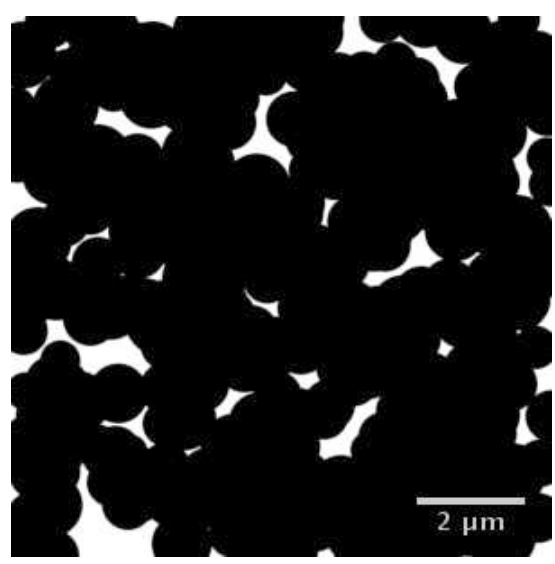

(b)

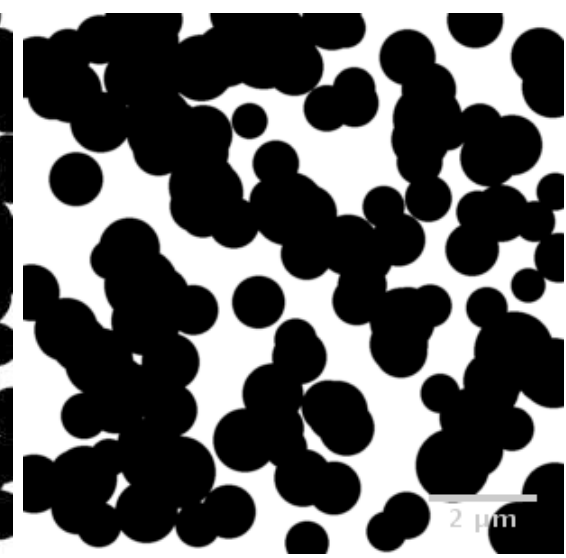

(c)

Figure 1: Neighborhood areas (in black) for different track lengths (a) 60 steps with the excluded volume fraction (EVF) of $95 \%$, (b) 180 steps with an EVF of $93 \%$ and 540 steps with an EVF of $65 \%$.

Table 1 gives an overview of the simulated models and the corresponding categories. The next step is to find some of those models in experimental video data using image processing and tracking algorithms.

\begin{tabular}{|c|c|c|c|}
\hline Model: & ND & CD & AD \\
\hline \hline Category: & Normal diffusion & Subdiffusion & Subdiffusion \\
\hline Model: & AT & ND + AT & CD + AT \\
\hline Category & Superdiffusion & Superdiffusion & Superdiffusion \\
\hline \multicolumn{4}{|c|}{ Table 1: Simulated models and their categories }
\end{tabular}

\subsection{Image processing and tracking}

Image processing and tracking were carried out with Fiji. ${ }^{12}$ Before tracking, we removed the background from each video. Therefore we estimate the minimum intensity for each pixel along the time axis because the particles are brighter than the background. Each frame was then divided by the estimated background. After that, a non-local means noise filter ${ }^{13,14}$ were applied to the video. Finally the trajectories were extracted by the Fiji plugin TrackMate ${ }^{15}$ which implements the robust tracking algorithm from Jaqaman. ${ }^{16}$

\subsection{Classification}

\subsubsection{Trajectory characterization by TrAJ}

TraJ $^{17}$ is a java library for simulation, analysis and characterization of $2 \mathrm{D}$ trajectories. Once trajectories were imported (from TrackMate or other tracking programs) or generated by TraJ, they can be characterized by numerous features. For the classification purposes of this work we used the following normalized features:

- Elongation (EL): Defined as $1-1 / \mathrm{R}$, where $\mathrm{R}$ is the ratio of the long side and short side of the minimum enclosing rectangle of the trajectory positions, the value ranges lies between 0 and 1 .

- Fractal dimension (FD): Estimates the fractal path dimension defined by Katz and George ${ }^{18}$ as $D=$ $\log (n) / \log (n d / L)$. Where $L$ is the total length of the path, $n$ is the total number of steps and $d$ is the largest distance between any two positions. For superdiffusion is takes values around 1-1.5, for normal diffusion it takes values of 1.5-2 and for subdiffusion it takes values larger than 2 .

- Long time / short time diffusion coefficient ratio (LTST-DC-RATIO): By evaluating the mean squared displacement (MSD) $\zeta^{2}(\delta)$ for different timelags $\delta=n \Delta t$ and fit a regression line to the data points it is possible to estimate the diffusion coefficient which is proportional to the slope of the regression line. By calculating the diffusion coefficient for different timelags it is also possible to estimate the diffusion coefficient for different time scales. Sub- and superdiffusion will lead to a lower or higher diffusion coefficient 
respectively on longer time scales. The short time diffusion coefficient $D_{\text {short }}$ is estimated by using the datapoints $\mathrm{n}=1$ to $\mathrm{n}=4$. Which datapoints are used for the long time diffusion coefficient $D_{\text {long }}$ depends on the length of trajectory $N_{t}$ : From $n=(K-3)$ to $K$ where $K=\left\lfloor N_{t} \cdot 0.1\right\rfloor$.

\subsubsection{Classification by random forests}

Random forests $(\mathrm{RF})$ is an ensemble based method which only uses an ensemble of decision trees. It was invented by Breiman and Cutler ${ }^{19}$ and combines bagging and random feature selection. Bagging generates different training samples by bootstrapping the original training data. Then, several decision trees are trained with randomly selected features. The predictions of those trees are combined into a single prediction by voting for the different prediction classes and then use the class with the most votes as prediction. For this work we use the randomForest package implemented in $\mathrm{R}$.

\section{RESULTS AND DISCUSSION}

\subsection{Classification of normal, sub- and superdiffusion}

For training the random forests we split the features (ED,FD,LTST-DC-RATIO) of simulated trajectories with a track length of 180 steps into two groups: $90 \%$ in a training group and $10 \%$ as testing group for evaluation purposes. A scatterplot matrix for the selected features EL, FD and LTST-DC-RATIO is shown in Figure 2. It can be seen that the fractal dimension allows a very good discrimination of the three diffusion classes. Furthermore the elongation and the LTST-DC-RATIO feature give strong indications for sub- and superdiffusion.

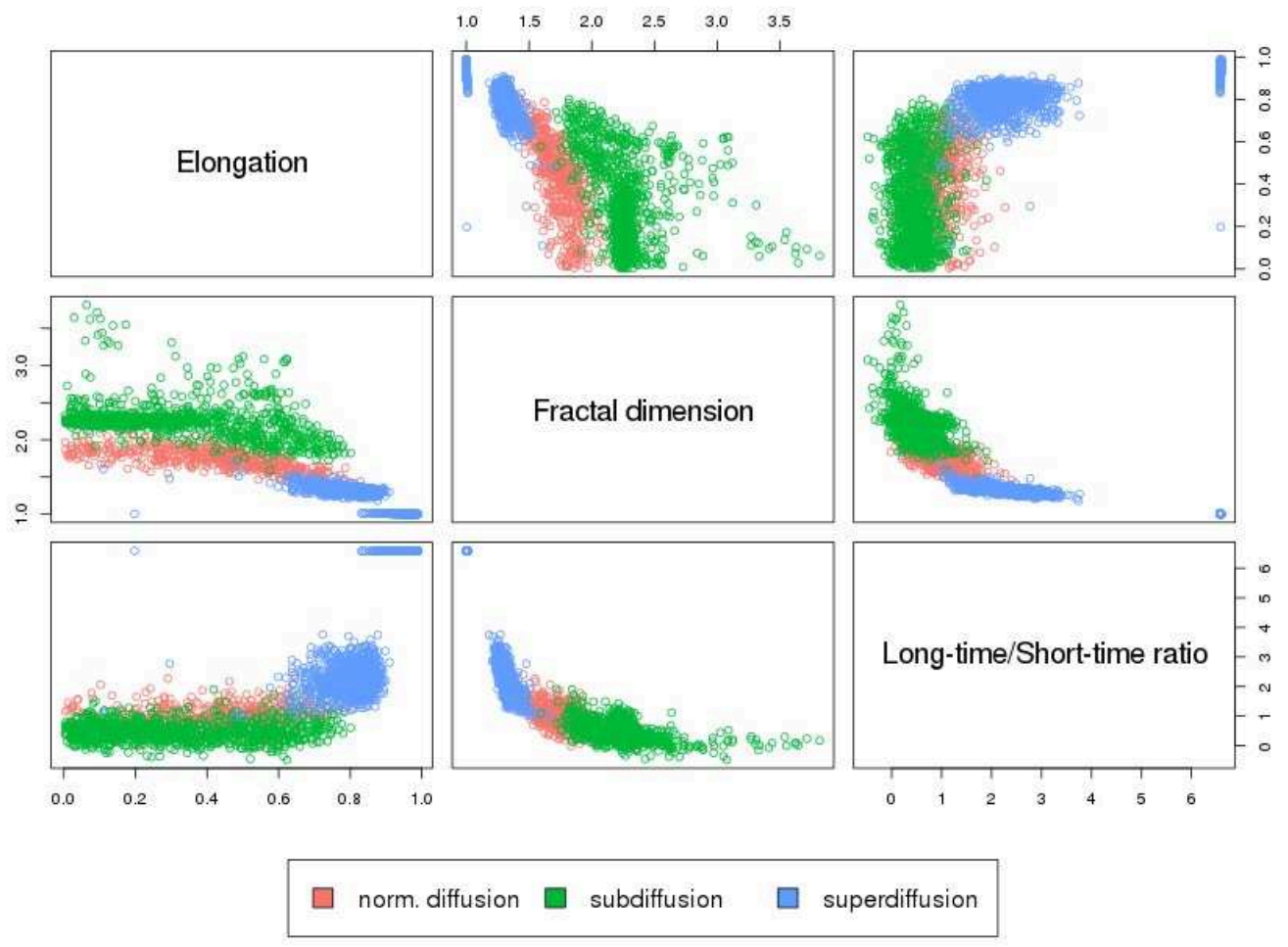

Figure 2: Scatterplot matrix of the three features EL, FD and LTST-DC-RATIO. Pairwise plotted data reveal distinct clusters for normal diffusion, sub- and superdiffusion.

After training we applied the classification for short, middle and long trajectories. Tables $2 \mathrm{~A}-\mathrm{C}$ show crosstabulations of expected and predicted classes for short, middle and long trajectories respectively. For short tracks, $40 \%$ of the trajectories which were actually sub- or superdiffusion were classified as normal diffusion. The reason for this error is likely the random nature of the process: The features display a huge spread for 


\begin{tabular}{|c|c|c|c|c|}
\hline \multicolumn{5}{|c|}{ A: Short trajectories } \\
\hline \hline pred. / exp. & Norm. & Sub. & Sup. & Class error \\
\hline Norm. & 48 & 10 & 23 & $40 \%$ \\
\hline Sub. & 3 & 96 & 1 & $4 \%$ \\
\hline Sup. & 4 & 0 & 115 & $3.3 \%$ \\
\hline
\end{tabular}

\begin{tabular}{|c|c|c|c|c|}
\hline \multicolumn{5}{|c|}{ B: Middle trajectories } \\
\hline \hline pred. / exp. & Norm. & Sub. & Sup. & Class error \\
\hline Norm. & 50 & 1 & 2 & $5.7 \%$ \\
\hline Sub. & 2 & 105 & 0 & $1.9 \%$ \\
\hline Sup. & 3 & 0 & 137 & $2.1 \%$ \\
\hline
\end{tabular}

\begin{tabular}{|c|c|c|c|c|}
\hline \multicolumn{5}{|c|}{ C: Long trajectories } \\
\hline \hline pred. / exp. & Norm. & Sub. & Sup. & Class error \\
\hline Norm. & 54 & 16 & 0 & $22 \%$ \\
\hline Sub. & 1 & 90 & 0 & $1.1 \%$ \\
\hline Sup. & 0 & 0 & 139 & $0 \%$ \\
\hline
\end{tabular}

\begin{tabular}{|c|c|c|c|c|}
\hline \multicolumn{5}{|c|}{ D: Performance measures } \\
\hline \hline & Short & Middle & Long & Overall \\
\hline Kappa & 0.7894 & 0.9574 & 0.9108 & 0.87 \\
\hline Accuracy & 0.8633 & 0.9733 & 0.9433 & 0.92 \\
\hline
\end{tabular}

Table 2: Confusion matrix for short (A), middle (B) and long (C) trajectories. The classes are normal diffusion (norm.), subdiffusion (sub.) and superdiffsion (sup.). Kappa and accuracy values for short, middle and long trajectories are shown in $(\mathrm{D})$.

short trajectories. However, if a track was classified as sub- or superdiffusion it was correct in more than $96 \%$ of all cases. For middle long trajectories table $2 \mathrm{~B}$ shows very low class error rates. For long tracks the class error for sub- and superdiffusion is very low. However, for normal diffusion the class error for normal diffusion is $22 \%$ because some of the subdiffusive tracks were classified as normal diffusion. In most cases the misclassified trajectories were of model class $\mathrm{AD}$ and not $\mathrm{CD}$ which both belong to the subdiffusion category. For a further evaluation of performance we calculated the accuracy which is defined as portion of correct classifications and additionally kappa statistic which takes into account that the classification could be correct for chance alone. For both performance measures the values range to maximum value of 1 . Table $2 \mathrm{D}$ summarizes the accuracy value and kappa statistic for short, middle and long trajectories. According to Altman ${ }^{20}$ the agreement between predicted and expected class could be rated as "very good" (Kappa: 0.81 - 1) for middle and long trajectories and as "good" (Kappa: 0.6-0.8) for short directories.

\subsection{Classification of experimental trajectories}

In the first approach, we used the method to classify the trajectories from the NTA device. From 87 trajectories with at least 60 steps, $78(89.6 \%)$ were classified as normal diffusion, $2(2.3 \%)$ were classified as subdiffusion and $7(8 \%)$ were classified as superdiffusion. That most of the tracks were classified as free diffusion trajectories confirmed our expectation. Even active convective transport or flow occurs sometimes in NTA experiments and the trajectories in the right column Figure 3 seems to be influenced by flow. However, subdiffusion should not occur in NTA derived trajectories. In those two cases, an evaluation of the probability for diffusion categories gave $40 \%$ for normal diffusion and $60 \%$ for subdiffusion, leading to incorrect classification. At least the experiment shows that our classification approach can reliably identify free diffusion. It is noteworthy that the classification
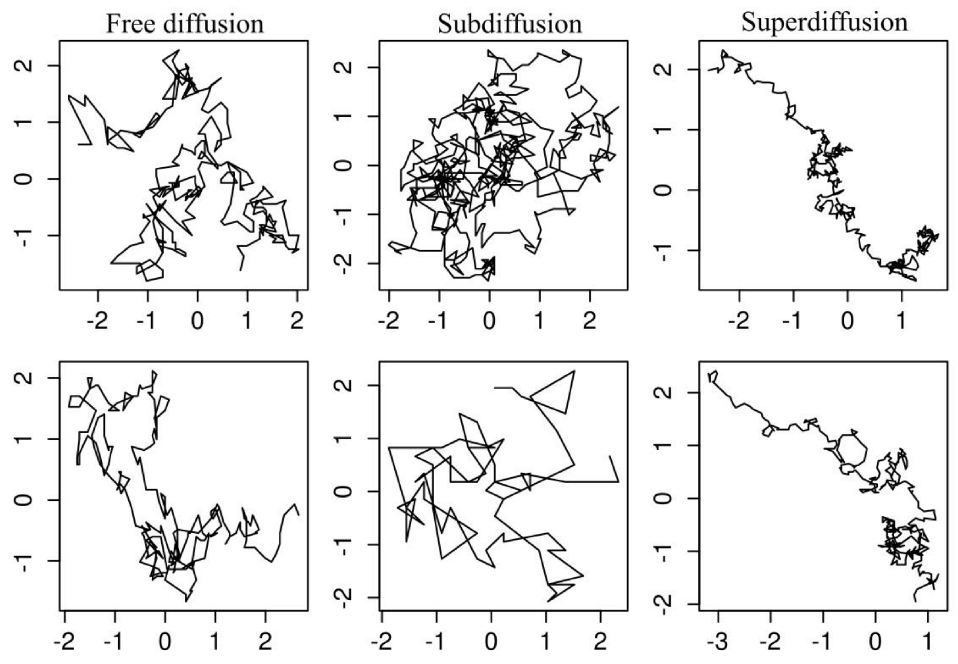

Figure 3: Example trajectories of $100 \mathrm{~nm}$ polystyrene captured by NTA technique for different diffusion categories. performance is independent from the diffusion coefficient and system camera properties since the random forests were trained with simulated trajectories for $50 \mathrm{~nm}$ particles at $22^{\circ} \mathrm{C}$, whereas the NTA experiments were carried out with $100 \mathrm{~nm}$ polystyrene particles. 

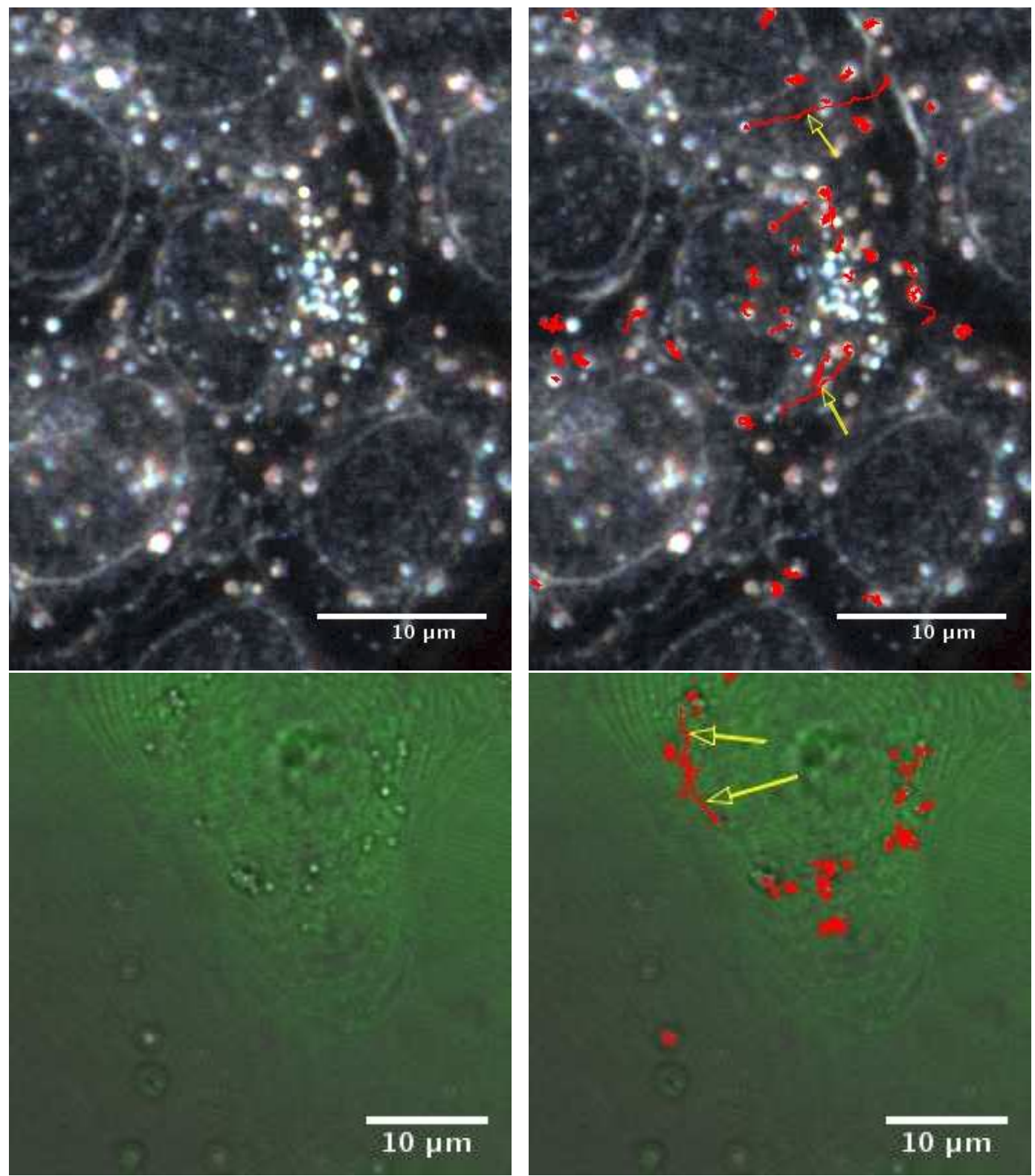

Figure 4: Single frames of video sequences showing gold nanoparticle containing V79 cells. Images from darkfield microscopy (upper panels) and confocal laser scanning microscopy (lower panels) with particles trajectories (red lines) recorded from the whole video sequence. Yellow arrows point to trajectories classified as active transports.

In the second approach we used video sequences of DFM and CLSM and analyzed a total of 81 and 72 trajectories, respectively. For the DFM trajectories, 73 were classified as subdiffusive, 6 as normal diffusion and 2 as super diffusion. In case of CLSM 66 trajectories were classified as subdiffusive, 4 as normal diffusion and 2 as superdiffusive. In both cases subdiffusion dominates the diffusion processes as expected. The classified active transports for DFM and CLSM were obviously correct and are labeled in figure 4.

However, at the moment the classifier is not applicable for trajectories that show different diffusion modes in a single trajectory. First tests suggest that it could be combined with a moving window approach but further investigations are necessary. 


\section{CONCLUSION}

In this study, we have shown that a random forests algorithm trained with the FD, EL and LTST-DC-RATIO successfully classifies trajectories into normal, sub- and superdiffusion. The new method was applied to nanoparticle trajectories of NTA, DFM and CLSM trajectories outside and inside cells. Simulation studies showed that its accuracy is "very good" for middle and long trajectories and still "good" for short trajectories. Some misclassifications may occur in case of sub- and superdiffusion, which were falsely classified as free diffusion. Finally it is worth to note that all datasets, java programs for simulation and $\mathrm{R}$ scripts for classification used for this work are open source and freely available at GitHub. ${ }^{10}$

\section{REFERENCES}

1. J. Mahowald, D. Arcizet, and D. Heinrich, "Impact of external stimuli and cell micro-architecture on intracellular transport states," ChemPhysChem, vol. 10, pp. 1559-1566, jul 2009.

2. N. Ruthardt, D. C. Lamb, and C. Bräuchle, "Single-particle tracking as a quantitative microscopy-based approach to unravel cell entry mechanisms of viruses and pharmaceutical nanoparticles," Mol Ther, vol. 19, pp. 1199-1211, jun 2011.

3. F. Persson, M. Lindén, C. Unoson, and J. Elf, "Extracting intracellular diffusive states and transition rates from single-molecule tracking data," Nature Methods, vol. 10, pp. 265-269, feb 2013.

4. G. Ruan, A. Agrawal, A. I. Marcus, and S. Nie, "Imaging and tracking of tat peptide-conjugated quantum dots in living cells: new insights into nanoparticle uptake, intracellular transport, and vesicle shedding," $J$. Am. Chem. Soc., vol. 129, pp. 14759-14766, nov 2007.

5. A. M. Bannunah, D. Vllasaliu, J. Lord, and S. Stolnik, "Mechanisms of nanoparticle internalization and transport across an intestinal epithelial cell model: Effect of size and surface charge," Mol. Pharmaceutics, vol. 11, pp. 4363-4373, dec 2014.

6. N. Monnier, S.-M. Guo, M. Mori, J. He, P. Lénárt, and M. Bathe, "Bayesian approach to MSD-based analysis of particle motion in live cells," Biophysical Journal, vol. 103, pp. 616-626, aug 2012.

7. D. Arcizet, B. Meier, E. Sackmann, J. O. Rädler, and D. Heinrich, "Temporal analysis of active and passive transport in living cells," Phys. Rev. Lett., vol. 101, dec 2008.

8. S. Huet, E. Karatekin, V. S. Tran, I. Fanget, S. Cribier, and J.-P. Henry, "Analysis of transient behavior in complex trajectories: Application to secretory vesicle dynamics," Biophysical Journal, vol. 91, pp. 35423559 , nov 2006.

9. J. Suh, K.-L. Choy, S. K. Lai, J. S. Suk, B. C. Tang, S. Prabhu, and J. Hanes, "Pegylation of nanoparticles improves their cytoplasmic transport," Int. J. Nanomed., vol. 2007, p. 735-741, Jan. 2008.

10. T. Wagner, "spie-photonics-europe-2016: Supplementary data," Zenodo, 2016, 10.5281/zenodo.46149.

11. X. Michalet, "Mean square displacement analysis of single-particle trajectories with localization error: Brownian motion in an isotropic medium," Physical Review E, vol. 82, oct 2010.

12. J. Schindelin, I. Arganda-Carreras, E. Frise, V. Kaynig, M. Longair, T. Pietzsch, S. Preibisch, C. Rueden, S. Saalfeld, B. Schmid, J.-Y. Tinevez, D. J. White, V. Hartenstein, K. Eliceiri, P. Tomancak, and A. Cardona, "Fiji: an open-source platform for biological-image analysis," Nature Methods, vol. 9, pp. 676-682, jun 2012.

13. A. Buades, B. Coll, and J.-M. Morel, "A non-local algorithm for image denoising," in 2005 IEEE Computer Society Conference on Computer Vision and Pattern Recognition (CVPR'05), Institute of Electrical \& Electronics Engineers (IEEE).

14. T. Wagner and P. Behnel, "ij-nl-means: Non local means 1.4.5," Zenodo, 2016, 10.5281/zenodo.45488.

15. J.-Y. Tinevez, J. Schindelin, C. Rueden, T. Wagner, T. Pietzsch, M. Hiner, S. Helfrich, C. Ye, and B. DeZonia, "Trackmate: 2.7.4-snapshot release," Zenodo, 2016, 10.5281/zenodo.45973.

16. K. Jaqaman, D. Loerke, M. Mettlen, H. Kuwata, S. Grinstein, S. L. Schmid, and G. Danuser, "Robust single-particle tracking in live-cell time-lapse sequences," Nature Methods, vol. 5, pp. 695-702, jul 2008.

17. T. Wagner, "Traj traj-0.2," Zenodo, 2016, 10.5281/zenodo.45639.

18. M. J. Katz and E. B. George, "Fractals and the analysis of growth paths," Bull. Math. Biol., vol. 47, no. 2, pp. 273-286, 1985.

19. L. Breiman, "Random forests," Machine Learning, vol. 45, no. 1, pp. 5-32, 2001.

20. D. G. Altman, Practical Statistics for Medical Research. Chapman and Hall/CRC, 1992. 